\title{
How planet-planet scattering can create high-inclination as well as long-period orbits
}

\author{
Sourav Chatterjee ${ }^{1}$, Eric B. Ford ${ }^{1}$ and Frederic A. Rasio ${ }^{2}$ \\ ${ }^{1}$ University of Florida, 211 Bryant Space Science Center, Florida, USA \\ email: s.chatterjee@astro.ufl.edu, eford@astro.ufl.edu \\ ${ }^{2}$ CIERA, Northwestern University, Evanston, IL 60208, USA \\ email: rasio@northwestern.edu
}

\begin{abstract}
Recent observations have revealed two new classes of planetary orbits. RossiterMclaughlin (RM) measurements have revealed hot Jupiters in high-obliquity orbits. In addition, direct-imaging has discovered giant planets at large $(\sim 100 \mathrm{AU})$ separations via direct-imaging technique. Simple-minded disk-migration scenarios are inconsistent with the high-inclination (and even retrograde) orbits as seen in recent RM measurements. Furthermore, forming giant planets at large semi-major axis $(a)$ may be challenging in the core-accretion paradigm. We perform many $N$-body simulations to explore the two above-mentioned orbital architectures. Planet-planet scattering in a multi-planet system can naturally excite orbital inclinations. Planets can also get scattered to large distances. Large- $a$ planetary orbits created from planet-planet scattering are expected to have high eccentricities $(e)$. Theoretical models predict that the observed long-period planets, such as Fomalhaut-b have moderate $e \approx 0.3$. Interestingly, these are also in systems with disks. We find that if a massive-enough outer disk is present, a scattered planet may be circularized at large $a$ via dynamical friction from the disk and repeated scattering of the disk particles.
\end{abstract}

Keywords. scattering, methods: n-body simulations, methods: numerical, planetary systems

\section{Introduction}

The 15 years since the discovery of the first exoplanet around a Solar-like star (Mayor \& Queloz 1995) have seen a revolution in our understanding of planet formation and evolution. Observations and theoretical modeling have worked hand-in-hand to discover and explain new architectures of planetary orbits. It is now well known that many exoplanets have large $e$ compared to our Solar system planetary orbits, indicating an active dynamical history (e.g., Chatterjee et al. 2008; Jurić \& Tremaine 2008; Nagasawa et al. 2008).

Recent RM measurements of many transiting planets are putting further constraints on theoretical models of various planet formation and evolution scenarios (e.g., Triaud et al. 2010; Winn et al. 2010; Morton \& Johnson 2011). Indeed, recent measurements find a large population of highly inclined planetary orbits, and even some retrograde orbits (see contributions from Winn et al. and Triaud et al. in this volume). Disk-planet migration models generally predict alignment between the planetary orbital angular momentum and the stellar spin axis from an aligned protoplanetary disk. Thus high-inclination orbits suggest dynamical evolution to be important in shaping the exoplanet architectures. Alternatively, inclined orbits might also result if the inner portion of the protoplanetary disk itself had been misaligned (Lai et al. 2011, see also Lai et al. in this volume).

Recent high-contrast imaging has revealed another class of planets - giant planets at very large $a$ ( $\gtrsim 50 \mathrm{AU}$; e.g., Kalas et al. 2008; Marois et al. 2008; Ducourant et al. 2008; 
Ireland et al. 2011). Timescale considerations for the core-accretion model of planet formation indicates that forming these planets in situ may be hard (e.g., Levison \& Stewart 2001; Dodson-Robinson et al. 2009). Instead, we consider formation at closer orbital separations followed by planet-planet scattering to launch planets in large- $a$ orbits from dynamically active systems (e.g. Chatterjee et al. 2008; Jurić \& Tremaine 2008; Nagasawa et al. 2008). However, these simulations predict that these orbits typically also have high $e \gtrsim 0.6$. Interestingly, some of the observed large- $a$ systems also have disks (e.g. Kalas et al. 2008) and dynamical modeling of these disks indicates moderate values of $e \approx 0.3$. We have started to explore the possibility that a planet launched into a large- $a$ and high-e orbit can later be circularized near its apocenter if the planet enters a debris disk during its apocenter excursion.

In Section 2 we summarize our numerical setup and present results for expected inclinations from planet-planet scattering. In Section 3 we discuss how planet-planet scattering followed by circularization due to a residual disk may create moderate- $e$ giant planets at large $a$. In Section 4 we conclude.

\section{Orbital inclinations from planet-planet scattering}
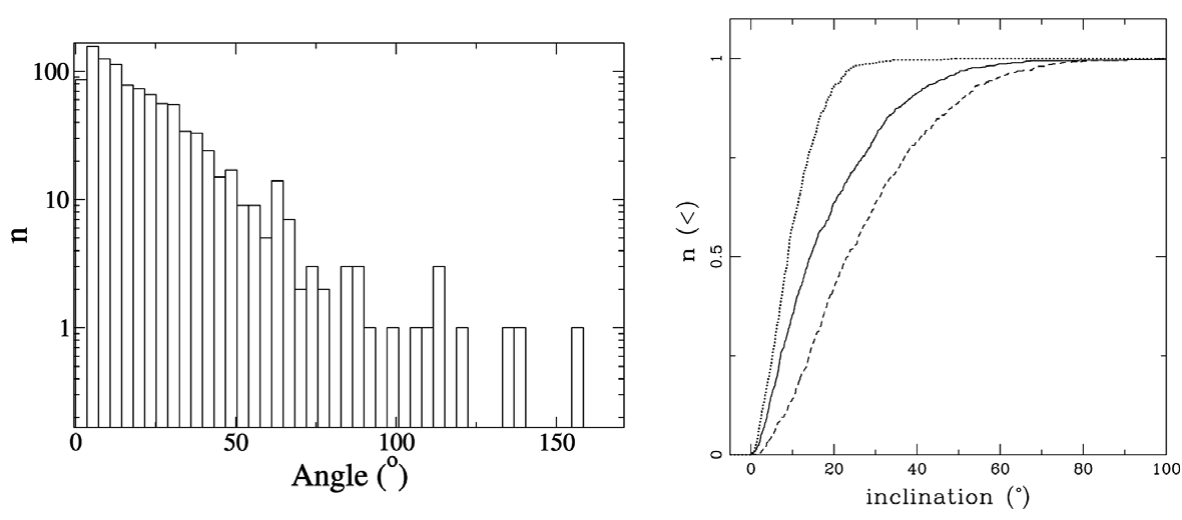

Figure 1. Distribution of orbital inclinations with respect to the initial invariable plane. Left: Histogram for the final inner-planet orbit. In our simulations about $2 \%$ of final inner planets are in retrograde orbits. Right: Cumulative histograms for the final inner (solid), and outer (dotted) planets. The relative inclinations between the inner and outer orbits (dashed) are also shown. About $20 \%$ of our simulated systems with two giant planets at the end have relative inclination angles $\geqslant 40^{\circ}$ and could later go through Kozai-type oscillations.

We have simulated 3 giant planets with masses between $0.4-1.2$ Jupitar-mass $\left(\mathrm{M}_{\mathrm{J}}\right)$ around a Solar mass star. The initial innermost planet is placed at $3 \mathrm{AU}$, and the other 2 planets are placed with planet-planet separation of 4.4 Hill radii. The initial $a$ 's were chosen to avoid Mean-motion resonances. The initial $e$ for the planetary orbits are chosen uniformly between $0-0.1$. Initial $i$ is chosen uniformly between $0^{\circ}-10^{\circ}$ with respect to the initial innermost orbital plane. All phase angles are assigned random values in the full range. Each of the above configurations is integrated using the hybrid integrator of MERCURY6.2 (Chambers 1999) for $10^{7} \mathrm{yr}$. If the energy conservation is poorer than $10^{-3}$ then the full integration is repeated using the Burlisch-Stoeer (B-S) integrator (see Chatterjee et al. 2008 for more details).

Figure 1 shows the distributions of the final inner- and outer-planet orbital inclinations with respect to the initial invariable plane, as well as the relative inclinations between 
the orbits. We find that planet-planet scattering is very efficient at exciting the inclination of planetary orbits. These high inclinations are not mere reflection of the initial inclinations. There is in fact no correlation between the initial and final inclinations. Starting from only moderate inclinations planet-planet scattering can create large inclinations extending all the way to retrograde orbits (although only about $2 \%$ in these simulations). The relative inclinations between the planetary orbits in systems where two giant planets remain bound are also high (median $\approx 25^{\circ}$ ). Note that the median value is incidentally in good agreement with the recent relative inclination measurement for $\nu$-Andromidae (c \& d, $29.9^{\circ} \pm 1^{\circ}$; McArthur et al. 2010). In our simulations about $20 \%$ systems show relative inclinations angles $\geqslant 40^{\circ}$, making them potentially Kozai active.

\section{Long-period planets}
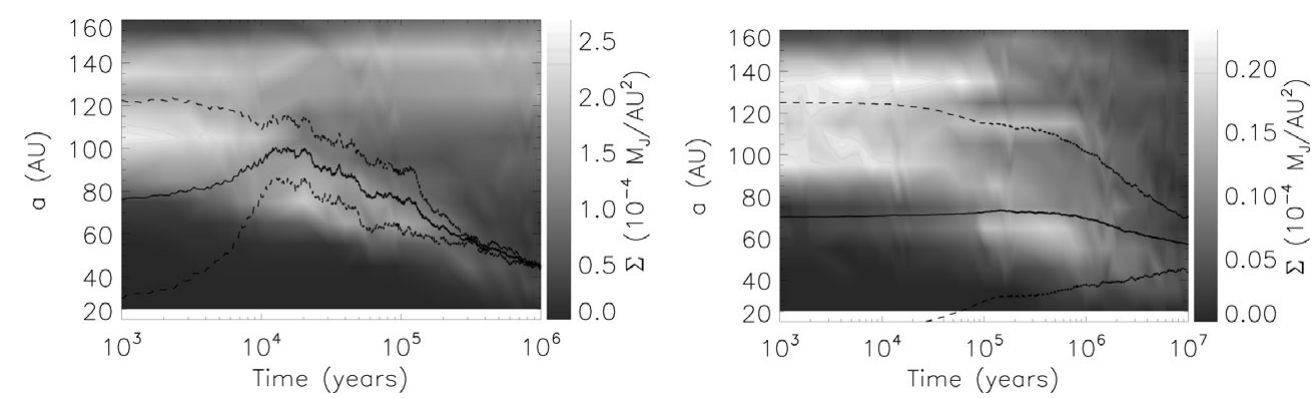

Figure 2. Evolution of the planetary orbit and surface density of the disk. Left: Evolution for the higher density disk model1. Right: Evolution for the lower density disk model2. The planets migrate outwards at first due to dynamical friction from the disk. The later inward migration of the planets is due to random outward scattering events of the disk particles by the planet. Planetary $e$ decreases throughout the entire evolution.

Planet-planet scattering naturally creates large- $a$ orbits. In our simulations with 3 giant planets we find outer planets with $a$ up to about 150 AU. Here we explore the following scenario. A massive disk of rocky material (possibly relic from the planet-formation process) remains at large separation from the star. This disk can remain relatively undisturbed for some time while the planets remain much closer to the star. At some point in the evolution, planet-planet scattering launches one of the planets into a large- $a$, and high-e orbit so that the giant planet enters the disk near apocenter. The Keplerian velocity of the planet near apocenter is less than that of the material in the disk as long as the $e$ of the planet is higher than the typical $e$ of the disk material. Thus, while in the disk the planet experiences a force due to dynamical friction from the disk in the direction of the planet's orbital velocity, increasing the planet's orbital energy and angular momentum. As a result, the planet's pericenter is raised as the orbit is circularized. The amount of migration and the timescale for circularization are directly proportional to the disk density.

Using the B-S integrator in MERCURY6.2 we simulate the evolution of a giant planet orbit initially in a large- $a$ and high- $e$ orbit (possibly created via a planet-planet scattering event). The initial $a$, and $e$ of the planetary orbit are $70 \mathrm{AU}$, and 0.7 , respectively. Note that, in this case our $t=0$ is after a planet-planet scattering event in a multi-giant planetary system that has launched a giant planet into this orbit. A disk of material is distributed initially between $90-150 \mathrm{AU}$ with a uniform surface density. The mass in 
the disk is represented in our simulations by $10^{3}$ equal-mass particles. The disk particles interact with the planet but not with each other. Initial $e$ and inclination of the disk material are chosen uniformly between $0-0.3$ and $0^{\circ}-4^{\circ}$, respectively. The planetary orbit is assumed to be aligned with the mid-plane of the disk (as a first step). We use two models varying the initial disk surface densities $(\Sigma)$ keeping everything else fixed. Our model1 and model2 have $\Sigma=10^{-4}$ and $10^{-5} \mathrm{M}_{\mathrm{J}} \mathrm{AU}^{-2}$, respectively.

Figure 2 shows the evolution of the planetary orbit as well as the surface density contours of the disk. For model1 (Figure 2, left), the planet first migrates outwards from $70 \mathrm{AU}$ to $\approx 100 \mathrm{AU}$ in $\approx 10^{4} \mathrm{yr}$. Note that the outward migration happens via circularization of the planetary orbit near the planet's apocenter. At the end of this migration the planet is in an orbit with $a=100 \mathrm{AU}$ and $e=0.14$. The outward migration is halted when the planet's intrusion severely depletes the disk. Until then the planet's evolution is dominated by dynamical friction arising from the disk. During this process the planet scatters a part of the disk material inwards (seen as a strip of over-density extending inwards). The subsequent evolution of the planetary orbit is governed by random scattering events between the planet and disk particles. The planet migrates inwards by scattering outwards disk particles that the planet had previously scattered inwards. This is a much slower process compared to the initial dynamical-friction dominated outward migration. The $e$ is damped throughout the entire process. At the integration stopping time $\left(10^{6} \mathrm{yr}\right)$ the planet is in an orbit with $a=44 \mathrm{AU}$ and $e=0.02$.

Qualitatively similar behavior is noted in the evolution of the planetary orbit for model2. However, in this case the timescale of the outward migration is about 10 times longer $\left(\sim 10^{5} \mathrm{yr}\right)$ and the magnitude of migration is less by a factor of 10 compared to model1. At the end of the dynamical-friction-driven outward migration the planetary orbit has $a=74 \mathrm{AU}$ and $e=0.5$. This stage of evolution is followed by the planet-disk particle scattering stage during which the planet migrates inwards. At the integration stopping time $\left(10^{7} \mathrm{yr}\right)$ for model2 the planetary orbit has $a=57 \mathrm{AU}$ and $e=0.2$.

In both cases at the end the planet is (or will be) left in a large- $a$, and modest- $e$ orbit. In addition, the total mass in the disk is reduced dramatically. The intrusion of the giant planet also excites the $e$ and inclination of the disk material. Furthermore, a low density disk may remain outside the planetary orbit which may then, over time, grind to create a dust ring via collisions, similar to the one observed in Fomalhaut-b.

\section{Discussion}

Using numerical simulations we have studied how planet-planet scattering can naturally create high-inclination orbits. We have studied whether giant planets can be launched into large- $a$, but modest-e orbits, similar in architecture to Fomalhaut-b, via planet-planet scattering. We find that planet-planet scattering can naturally create many large- $a$ orbits, however, these orbits are also expected to have high $e$ (e.g., Chatterjee et al. 2008). Nevertheless, if a massive outer disk exists and the scattered giant planet near its apocenter enters the disk, dynamical friction from the disk can raise the planet's $a$ until the planet reduces the disk density significantly via scattering. Then the planet can migrate inwards via scattering some of the remaining disk particles outwards. During the whole process the $e$ of the planetary orbit reduces. We plan to further study this process in detail exploring different disk masses, densities, as well as varying planet masses. We thank the SOC and LOC for arranging this excellent conference and the opportunity to present these results. 


\section{References}

Chambers, J. E. 1999, MNRAS, 304, 793

Chatterjee, S., Ford, E. B., Matsumura, S., \& Rasio, F. A. 2008, ApJ, 686, 580

Dodson-Robinson, S. E., Veras, D., Ford, E. B., \& Beichman, C. A. 2009, ApJ, 707, 79

Ducourant, C., et al. 2008, A\&BA, 477, L1

Ireland, M. J., et al. 2011, ApJ, 726, id.113

Jurić, M. \& Tremaine, S. 2008, ApJ, 686, 603

Kalas, P., et al. 2008, Science, 322, 1345

Lai, D., Foucart, F., \& Lin, D. N. C. 2011, MNRAS, 412, 2790

Levison, H. F. \& Stewart, G. R. 2001, Icarus, 153, 224

Marois, C., et al. 2008, Science, 322, 1348

Mayor, M. \& Queloz, D. 1995, Nature, 378, 355

McArthur, B. E., et al. 2010, ApJ, 715, 1203

Morton, T. D. \& Johnson, J. A. 2011, ApJ, 729, id.138

Nagasawa, M., Ida, S., \& Bessho, T. 2008, ApJ, 678, 498

Triaud, A. H. M. J., et al. 2010, A\&AA, 524, A25

Winn, J. N., Fabrycky, D., Albrecht, S., \& Johnson, J. A. 2010, ApJ, 718, L145 